\title{
The Influence of Compressive Stresses on the Properties of Inductive Electronics Components
}

\author{
J. SALACH ${ }^{a, *}$ AND P. NOWAK ${ }^{b}$ \\ ${ }^{a}$ Institute of Metrology and Biomedical Engineering, Warsaw University of Technology, \\ św. A. Boboli 8, 02-525 Warsaw, Poland \\ ${ }^{b}$ Industrial Research Institute for Automation and Measurements PIAP, \\ Al. Jerozolimskie 202, 02-486 Warsaw, Poland
}

\begin{abstract}
This paper presents results of investigation of stress influence on magnetic characteristics of ferrite ring cores for inductive electronics components. Ring-shaped cores were made of $\mathrm{Ni}-\mathrm{Zn}$ ferrite designed for anti-lung filter cores. The results of the study indicate a significant impact of external forces stresses on the magnetic properties of ferrite cores. Such changes are very important due to their influence on the correct operation of the induction components.
\end{abstract}

DOI: 10.12693 /APhysPolA.133.1053

PACS/topics: $75.50 . \mathrm{Gg}, 75.30 .-\mathrm{m}$

\section{Introduction}

The cores of inductive components are crucial element of these devices. These are usually inductors, transformers, or electromagnets, usually used as wounded ring cores made of metaloxide ceramic. The quality, precision, and stability of the entire device depends mostly on the cores' parameters. Their characteristics may be influenced by a lot of factors, such as temperature, humidity, and stresses. The strains can have two sources: core's internal stresses and external forces. Internal stresses are associated with the manufacturing process, mostly caused by the residual stresses in the core or too tight winding, and should be eliminated during the production of cores. On the other hand, external stresses can occur due to an improper installation, or other incidental factors which apply a force to the core.

The most important thing, from the users and constructors the point of view, is the knowledge about the stresses' effects on the properties of the magnetic cores of an inductive component. The impact on the ferrites in the form of frame cores has been previously reported [1]. However, so far no research have confirmed a clear impact of stresses on the ring-shaped core. The results of such research are presented in the paper. The tests were conducted in a way which ensured both the uniformity of stress distribution in the core as well as closed magnetic path. This methodology has been described for amorphous cores ring in previous publications [2], and was adjusted to the ceramic ferrite cores tests.

\section{Methodology of investigation}

The construction of the inductive electronic components can be divided for two distinct groups of elements.

${ }^{*}$ corresponding author; e-mail: j.salach@mchtr.pw.edu.pl
These are the inductive coils (with magnetic core, mostly often ring-shaped, with mechanical elements for bounding) and electrical transformers. These elements are widely available and used for the construction of electronic systems such as filter systems, separation systems, resonator systems, etc. The main element, that has the greatest impact on their utility parameters such as the nominal value and the tolerance to which they characterize, is the magnetic core. This core is most often made of ceramic magnetite - ferrite. The influence of temperature on the parameters of the induction cores of electronic components is well known, but the influence of stress on their properties is not as obvious.

The main objectives which should be fulfilled by the test stand for this type of cores are:

- enable application of uniform compressive and tensile stresses to the core. When distribution of stresses is non-uniform, such as in the case of ring-shaped magnetoelastic sensors developed by Mohri [1, 2], results of investigation are difficult for interpretation from physical point of view. Moreover, the range of possible stresses is limited to the stress strength of the most stressed area of the core,

- enable utilization of cores with closed magnetic circuit, such as ring-shaped cores. When core's magnetic circuit is open, demagnetization occurs. In such a case interpretation of results of investigation requires analysis of demagnetization, which is non-linear and difficult to determine with sufficient accuracy [3], especially in the case of high permeability materials,

- utilize magnetic materials cores of shapes used for industrial applications. Closed circuit facilitates analysis of received results. In some cases the closed circuit in general allows for the measurement to be made [4].

The idea of the method for applying compressive stresses to the ring-shaped core made of ceramic magnetic material is presented in Fig. 1. Compressive force is applied perpendicularly to the base of ring-shaped core. 


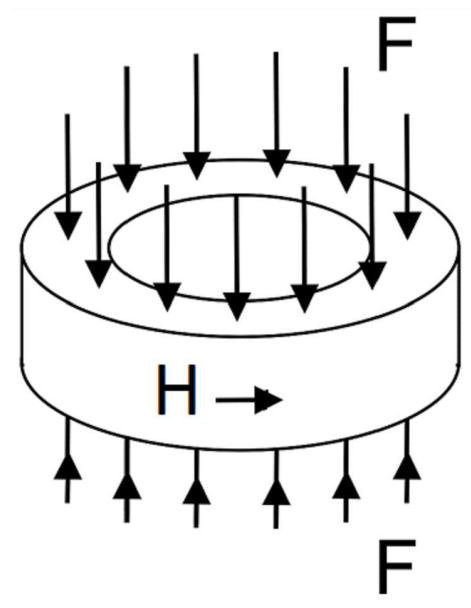

Fig. 1. Idea of the method of application uniforms stresses from external force $F$ to the ring-shaped core. $H$ presents the direction of applied magnetic field.

As a result, uniform distribution of compressive stresses is assumed in the tested core. However, it should be noted that the direction of stresses is perpendicular to the direction of magnetizing field, which should be considered in interpretation of experimental results.

Technical solution of mechanical system enabling application compressive stresses to the ring-shaped core is presented in Fig. 2.

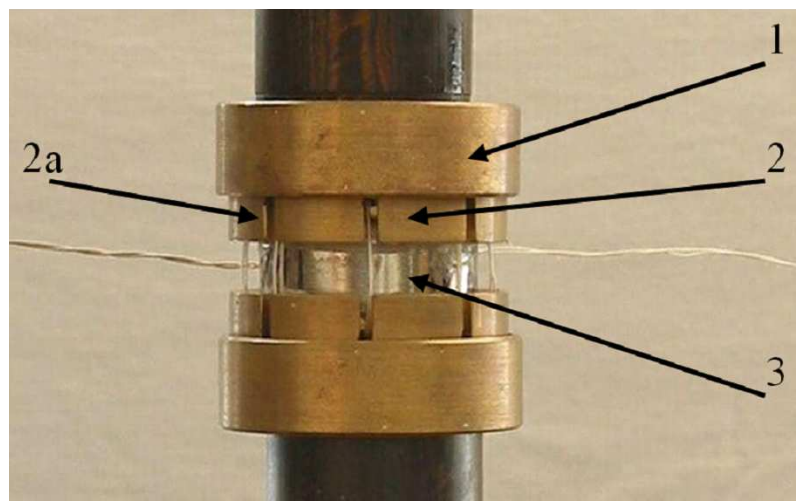

Fig. 2. Mechanical system for application of the uniform stresses to ring-shaped core compressive stresses: 1 - base backings, 2 - cylindrical, non-magnetic backings, $2 \mathrm{a}$ - grooves for the windings, 3 - tested core.

In the case of compressive stresses [5] a special set of nonmagnetic, cylindrical backings was used, in order to obtain the uniform compressive stresses in the tested ring-shaped core and enable it to be wound. Between the nonmagnetic backing (2) and the ring core (3), a special elastic spacer is placed, to guarantee the uniform distribution of the stresses in the tested core (3). Magnetizing and measuring windings are placed in a special grooved races $(2 \mathrm{a})$ in backings. Compressive force is applied to device by hydraulic press, via the base backings (1). The ball joint is used to avoid bending of the sample, which could lead to a non-uniform stress distribution in the sample.

For investigation focused on compressive stresses ringshaped sample made of $\mathrm{Ni}-\mathrm{Zn}$ ferrite was selected. This material has high permeability (around 6000 ) and negligible magnetostriction. The outside diameter of the core was $26 \mathrm{~mm}$, inside diameter was $16 \mathrm{~mm}$ and core's height was $15 \mathrm{~mm}$. Magnetic hysteresis loops were measured by digitally controlled hysteresis graph HB-PL3.0.

Magnetizing field frequency was $0.5 \mathrm{~Hz}$ which enables quasi-static measurements. HBPL hysteresis graph consists of precise function generator, voltage-to-current converter as well as ultrastable integrator. Before each measurement, the drift compensation of the integrator as well as sample demagnetization were performed.

\section{Results of investigation}

Results of research show the significant influence of compressive stresses on the magnetic properties of $\mathrm{Ni}-\mathrm{Zn}$ ferrite cores. Obtained results are presented in Figs. 3-6.

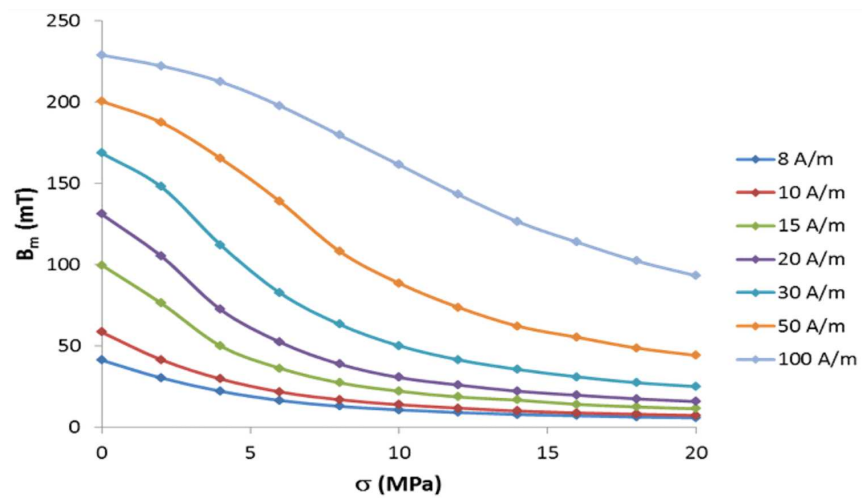

Fig. 3. Influence of compressive stresses $\sigma$ on maximal value of flux density $B$ achieved for different values of amplitude of magnetizing field $H_{m}$ in ring-shaped core made of $\mathrm{Ni}-\mathrm{Zn}$ ferrite. Values of amplitude of magnetizing field $H_{m}$ are multiples of coercive field.

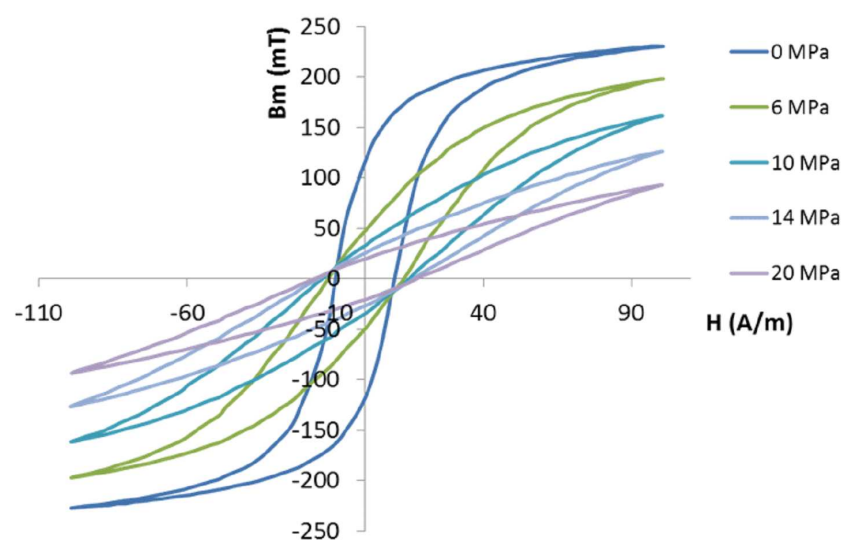

Fig. 4. Influence of compressive stresses $\sigma$ on hysteresis loop $B(H)$ in ring-shaped core made of $\mathrm{Ni}-\mathrm{Zn}$ ferrite. 


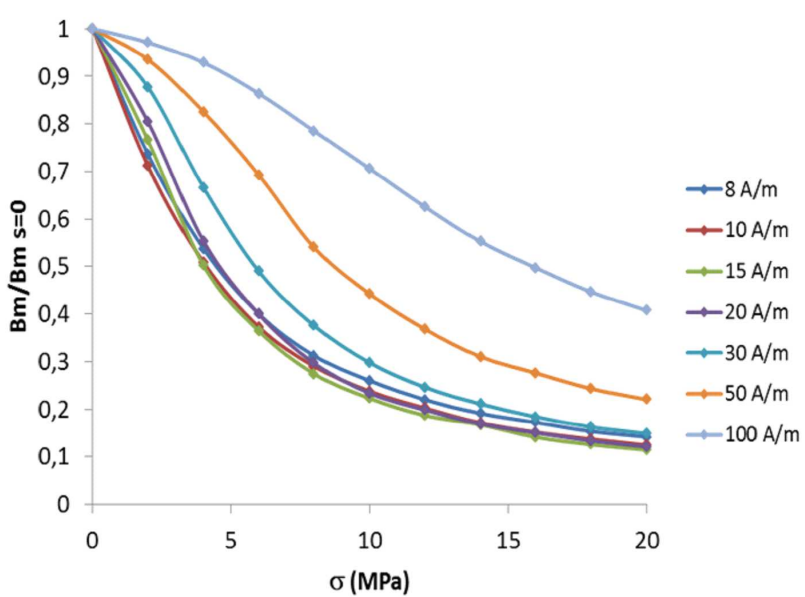

Fig. 5. Influence of compressive stresses $\sigma$ on maximal value of flux density $B$ (arbitrary unit) achieved for different values of amplitude of magnetizing field $H_{m}$ in ring-shaped core made of $\mathrm{Ni}-\mathrm{Zn}$ ferrite. Values of amplitude of magnetizing field $H_{m}$ are multiples of coercive field.

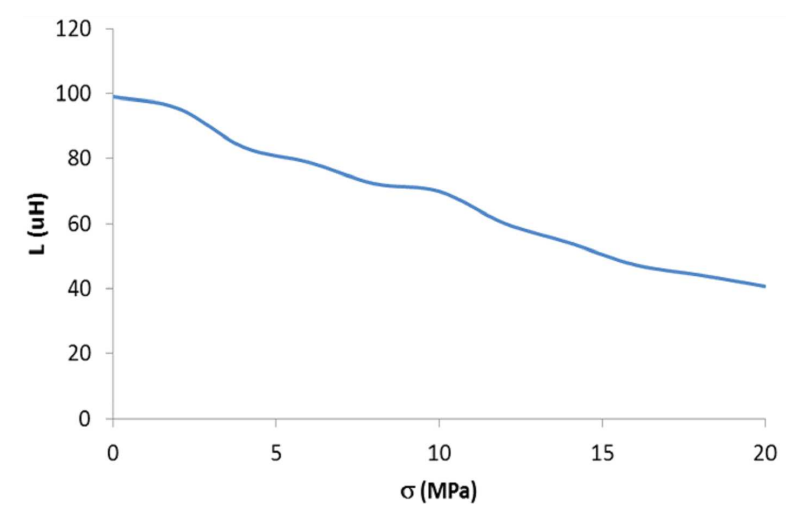

Fig. 6. Influence of compressive stresses $\sigma$ on inductance $L$ in $\mathrm{Ni}-\mathrm{Zn}$ ferrite.
The results of investigation showed a significant influence of compressive stress $\sigma$ on the value of the flux density $B$ in $\mathrm{Ni}-\mathrm{Zn}$ ferrite cores. This influence is particularly evident for those values expressed in arbitrary units. The changes are significant and they reach $90 \%$.

The tests performed on the inductor also show significant changes in its inductance $L$ (Fig. 6).

\section{Conclusion}

The study showed that the effect of stress on the magnetic properties of ferrite ring cores is significant. The compressive stresses have a much greater influence on the magnetic properties of the core for lower values of magnetizing field.

The results show that the compressive stresses can cause significant changes introduced by inductive components with ferrite cores.

\section{References}

[1] A.E. Clark, M. Wun-Fogle, IEEE Trans. Magn. 25, 3611 (1989).

[2] K. Mohri, S. Korekoda, IEEE Trans. Magn. 14, 1071 (1978).

[3] A. Bienkowski, R. Szewczyk, J. Salach, Polish Patent PAT.210004 2007; M. Sablik, S. Rubin, L. Rilez, D. Jiles, D. Kaminski, S. Biner, J. Appl. Phys. 74, 480 (1993).

[4] D. Jackiewicz, R. Szewczyk, A. Bienkowski, J. Electr. Eng. Slovak 66, 178 (2015); D. Jackiewicz, M. Kachniarz, A. Bieńkowski, in: Proc. of the 15th International Conference on Global Research and Education Inter-Academia 2016, Eds. R. Jablonski, R. Szewczyk, Berlin 2017, p. 63.

[5] A. Bienkowski, R. Szewczyk, Polish Patent PAT.197641 2001. 